\title{
The Receiver Circuit of Visible Light Communication
}

\author{
Yuhe $\operatorname{Tian}^{1, \text { a }}$ \\ ${ }^{1}$ North China Electric Power University, Baoding 071000, China \\ atyhstudy@163.com
}

Keywords: visible light communication

\begin{abstract}
LEDs are semiconductor devices that emit light when biased in the forward direction of the p-n junction. LEDs present many advantages over traditional light sources, including lower energy consumption, longer lifetime, improved robustness and smaller size. A significant attribute of LEDs is their ability to switch on and off thousands of times per second. No other lighting technology has this capability. This switching occurs at ultra-high speeds, so far beyond what the human eye can detect, that the light appears to be constantly on. These embedded signals are emitted from the LEDs in the form of binary code; 'off' equals zero and 'on'equals one. This is what they call visible light communication (VLC). When VLC equipment and devices are placed throughout a building of geographical area,a comprehensive wireless communication network can be created.

This thesis talks about a stand alone receiver .The receiver will be a device to plug in a digital port of the computer. It receives information from the emitted light and send it to the PC.The transmitted information will give the receiver the possibility to determine his current position in a room by looking at the amplitude of the signals he receives from multiple LEDs. It can also be used to transmit other digital information/files.
\end{abstract}

\section{Introduction}

If we want to know the bits that are transmitted, we have to use several blocks. First of all, we will use a current-voltage converter, so that the voltage is also proportional to the incoming light. We also need a high pass filter to filter out the ambient light and the $100 \mathrm{~Hz}$ frequency components coming from the lighting in the building. Since the signal we will receive is weak, we will have to use an amplifier. Then, we also need an ADC to digitalize the received signal. Before the ADC, an anti-aliasing filter is needed. Finally, the digital samples have to be transmitted to a PC so we can use software to make some calculations and to find the position.

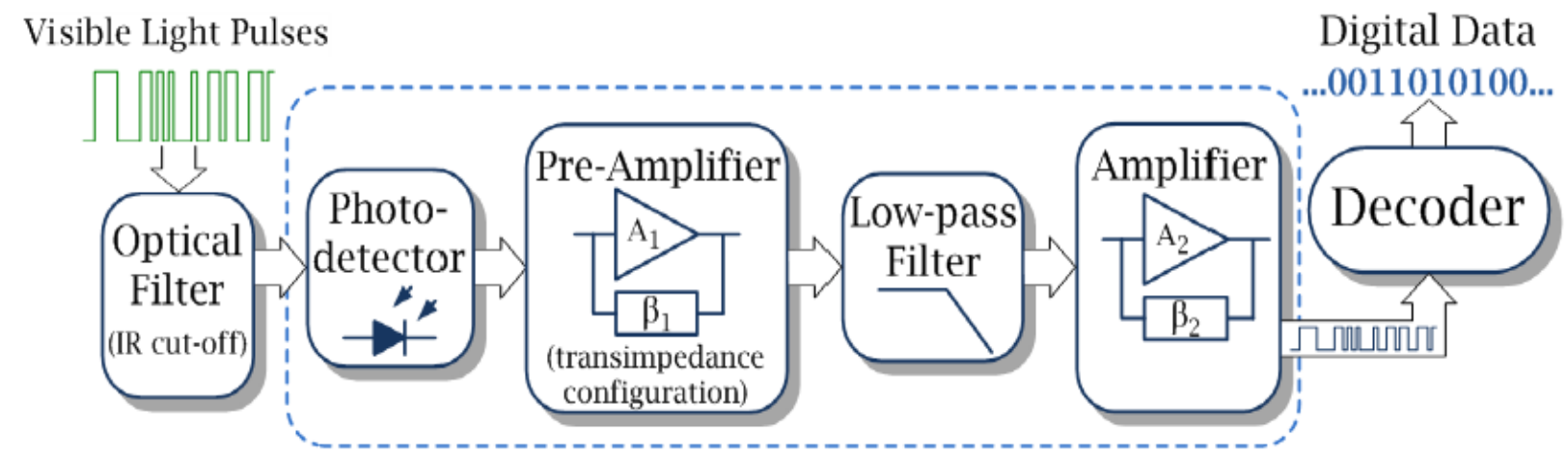

Figure1: VLC receiver block diagram

\section{Choice of receiving photodiode}

The first part of a VLC receiver, is a photosensitive element. Mostly, a photodiode is used at the input of the receiver. The current through the photodiode, is proportional to the light falling on it. The more light it receives, the larger the current will be.The photodiode has to operate in the same spectrum as the LED.We comparsioned between the PIN photodiode and the Avalanche photodiode .

Table1. Comparsion between the PIN photodiode and the Avalanche photodiode 


\begin{tabular}{lll}
\hline \multicolumn{1}{c}{ characteristic } & \multicolumn{1}{c}{ PIN Photodiode } & \multicolumn{1}{c}{ Avalanche Photodiode } \\
\hline Modulation bandwidth & Dozens MHZ dozens GHZ & Hundreds MHZ Hundreds GHZ \\
$\begin{array}{lll}\text { Optical current gain } \\
\text { Special circuit requirements }\end{array}$ & 1 & $\begin{array}{l}\text { None } \\
\text { High bias voltage and } \\
\text { temperature compensation } \\
\text { circuit }\end{array}$ \\
Linearity & High & $\begin{array}{l}\text { Low,Suitable for digital } \\
\text { applications }\end{array}$ \\
cost & Low & Middle to high \\
\hline
\end{tabular}

With reference to the parameters given in Table 1, due to the low cost of the application of the PIN photodiode, the modulation loan also meets the requirements of the indoor visible system, the most common of the visible light communication systems is the use of a PIN photodiode as a visible light receiver.

\section{Power supply}

We can use a step-up converter (boost converter) to obtain a larger voltage. There are several good chips you can use for this purpose. We found a circuit to boost the $1.2 \mathrm{~V}$ to3.3 $\mathrm{V}$ in the datasheet of the MCP 1640 of Microchip 1. This 3.3V can be enough to supply the circuit. You can see the circuit of the step-up converter in figure 4.2.

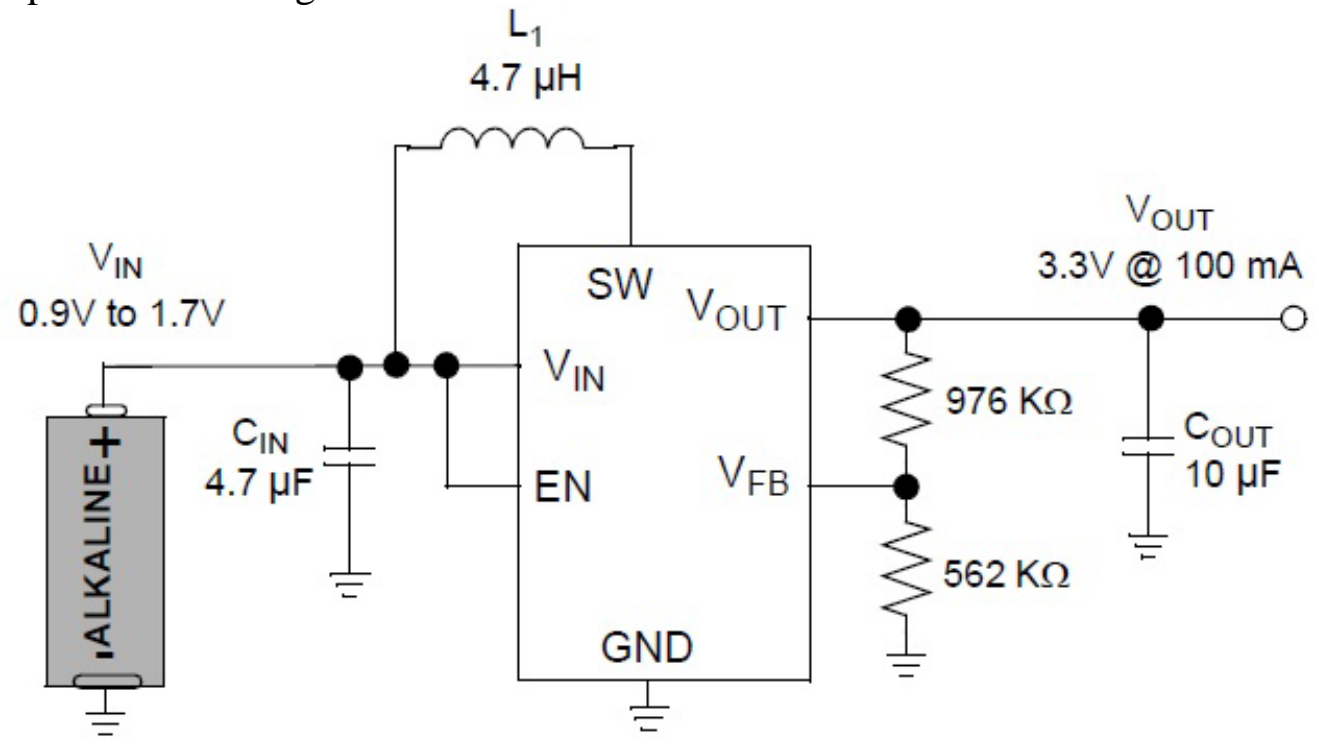

Figure 2: Step-up converter

\section{Current-voltage converter}

To convert the current from the photodiode in a voltage, we have to use a current-voltage converter. A common way to do this, is to use an op-amp for it, as shown in figure 3. 


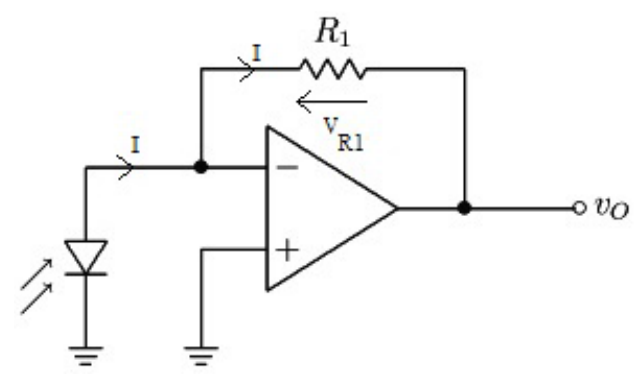

Figure 3: Current-voltage converter

\section{Amplifier}

Since the signal is too weak after filtering, we will use an amplifier. The amplification is needed if we want to use the full range of the ADC. After some measurements, we took an inverting op-amp with a gain of 100. Before the amplifier we put a high pass filter with $f_{\text {cut-off }}=\frac{1}{2 \cdot \pi \cdot 5100 \cdot 10 \cdot 10^{-9}}=31.2 \mathrm{HZ}$ and a gain of 1 . We did this because we still had an offset after the ambient light rejection circuit. We also tried to use two op-amp stages with each a gain of 10, but the results weren't so good. We still had an offset and there was more noise in the signal. So, we decided to use a high pass filter with a gain of 1 and an inverting amplifier with a gain of 100 . When we simulated the ambient light rejection circuit, no offset was visible. We just had a DC offset of 2.5 volt because we are working with a virtual ground. We also tried to build a high pass filter with a gain of 100, but an offset was still visible.

\section{Low-Pass filter}

In order to reduce signal noise and achieve a linear response in the pass-band, a Butterworth response is the most adequate. This filter type presents a practically flat response in the pass-band, with almost no ripple. At the projected cut-off frequency the attenuation is $3 \mathrm{~dB}$ and, for higher frequencies, it decreases with a steep $20 \mathrm{~dB}$ per decade per pole ratio. A multiple-feedback (MFB) configuration was chosen due to its low sensitivity to component variations and gain accuracy.

After selecting the necessary filter and circuit types, a $50 \mathrm{MHz}$ low-pass, unity-gain filter with 2 poles was chosen. The E24 series resistors and E12 series capacitors were selected and optional entries adjusted. The resulting circuit was then simulated in PSpice with a TEXAS OPA820ID operational amplifier. Also, a buffer was added between the preamplifier and the low-pass filter in order to increase the input impedance of the filter stage, thus not causing any effect on the preamplifier. Figure 4 represents the low-pass filter design with an input buffer.

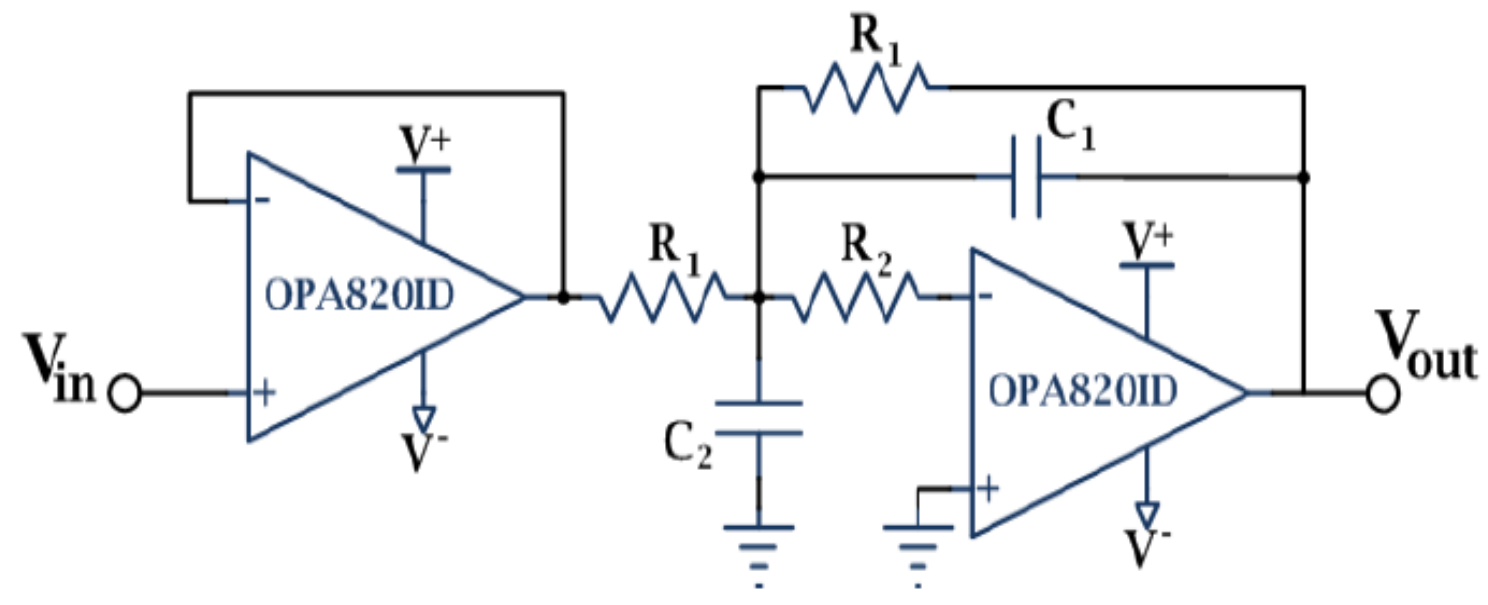

Figure 4: Low-pass filter with an input buffer 


\section{Conclusions}

Upon the conclusion of the work that served as the basis for the current text, several conclusions need to be registered. Starting with the research made on VLC systems, it appears to be a viable technology and has a good opportunity towards becoming a widespread technology in the future of optical wireless communications. This is also supported with the results obtained. However, there are still many things that need to be more thoroughly studied and analyzed.

An important constraint to VLC and this work as well, is the fact that manufacturers have not realized the potential in this type of applications. They can provide, in a near future, a new range of applications for their products. But in order for this commercial growth to happen, manufacturers need to release more information about their products. Although optical characteristics are well specified, in most datasheets the electrical transient behavior of the devices is not provided. This happens particularly in LED' s datasheet, making research of devices for a VLC transceiver a hard task.

Another important conclusion comes from the practical implementation of the designed devices. Although the experimental results of the projected devices are within the range of the simulations, it was left clear that prototyping is important. The behavior of the optoelectronic components cannot be fully translated into the simulations. Therefore, the best way to completely characterize the implemented devices is by prototyping, which becomes essential towards assessing their real behavior.

\section{References}

[1] Nuno Rafael,Mendonça Lourenço.Sistemas de Comunicação com Luz Visível:Emissor/Receptor .

Universidade de Aveiro:Departamento de Electrónica, Telecomunicações e Informática,2009.

[2] Jelle Smets, Rob Holvoet.Mains driven transmitter \& portable receiver for visible light communication Indoor positioning.Electrical Engineering Department at HES-SO: Syst emes industriels, Mr. Franc, ois Corthay Academic year: 2012 - 2013 